\title{
Computer Based Modeling of Vegetation Propagation across a Disturbed Space Linked to GIS
}

\author{
Lubos Matejicek $^{1}$ and Pavel Kovar ${ }^{2}$ \\ 1. Institute for Environmental Studies, Charles University, Benatska 2, Prague 128 01, Czech Republic \\ 2. Department of Botany, Charles University, Benatska 2, Prague 128 01, Czech Republic
}

\begin{abstract}
The paper is focused on computer simulation of natural vegetation propagation across two selected disturbed sites. Two sites located in the different environments, the abandoned sedimentation basin of a former pyrite ore mine and the ash deposits of a power station, were selected to illustrate the proposed spatio-temporal model. Aerial images assisted in identifying and monitoring the progress in the propagation of vegetation. Analysis of the aerial images was based on varying vegetation coverage explored by classification algorithms. A new approach is proposed entailing coupling of a local dynamic model and a spatial model for vegetation propagation. The local dynamic model describes vegetation growth using a logistic growth approach based on delayed variables. Vegetation propagation is described by rules related to seed and its dispersal phenomena on a local scale and on the scale of outlying spreading. The disturbed sites are divided into a grid of microsites. Each microsite is represented by a $5 \mathrm{~m} \times 5 \mathrm{~m}$ square. A state variable in each microsite indicates the relative vegetation density on a scale from 0 (no vegetation) to 1 (long-term maximum of vegetation density). Growth, local vegetation propagation and the effects of outlying vegetation propagation in each cell are described by an ordinary differential equation with delayed state variables. The grid of cells forms a set of ordinary differential equations. The abandoned sedimentation basin and the ash deposits are represented by grids of $185 \times 345$ and $212 \times 266$ cells, respectively. A few case-oriented studies are provided to show various predictions of vegetation propagation across two selected disturbed sites. The first case study simulates vegetation growing without spatial propagations and delayed variables in the spatio-temporal model. The second and the third case studies extend the previous study by including local and outlying vegetation propagation, respectively. The fourth case study explores delayed impacts in the logistic growth term and the delayed outcome by vegetation propagation across the disturbed space. The performed case-oriented studies confirm the applicability of the proposed spatio-temporal model to predict vegetation propagation in short-term successions and to estimate approximate vegetation changes in long-term development. As a result, it can be concluded that remotely sensed data are a valuable source of information for estimates of model parameters and provide an effective method for monitoring the progress of vegetation propagation across the selected sites, spaces disturbed by human activities.
\end{abstract}

Key words: Spatio-temporal modelling, vegetation propagation, disturbed space, remote sensing, GIS.

\section{Introduction}

The former mining area and the disposal sites of a thermal power station were used for exploration of primary vegetation development in the framework of long-term natural remediation. In order to describe the spatio-temporal propagation, a spatially explicit model for discrete space and continuous time can be used to perform simulation case studies for selected disturbed sites [1-3]. In addition to the models based on a set of partial differential equations or ordinary differential

Corresponding author: Lubos Matejicek, research fields: environmental modeling, GIS and remote sensing. equations, many mathematical techniques have been proposed, such as the finite-elements method, models based on fractal geometry, the catastrophes theory and cellular automata [4, 5]. Novel approaches have appeared during the last few decades, opening the way to some new aspects of research: in particular, classification methods in remote sensing for evaluation of land cover changes, spatial data management in GISs (Geographic Information Systems) for integration of spatio-temporal data, and a GPS (Global Positioning System) for spatially related observations in the field $[6,7]$. At the present time, 
GIS can be used to perform nearly all tasks focused on the spatio-temporal modeling of vegetation propagation. For time-consuming numerical simulations, it is necessary to couple GIS with advanced software tools designed for high performance in dynamic modeling. However, with the increasing power of GIS tools, this relationship needs to be reconsidered [8].

This paper describes a new approach for studying spatio-temporal dynamic systems focused on modeling of vegetation propagation across a disturbed space linked to GIS and simulation tools designed for high performance in dynamic modeling. Two sites located in different environments, the abandoned sedimentation basin of a former pyrite ore mine and the ash deposits of a power station [9], were selected to illustrate a proposed spatio-temporal model using selected case-oriented studies. Vegetation propagation across the disturbed space is considered in the Central European climatic environment. In the first stage, the varying vegetation coverage is explored by classification of aerial images. Images captured in 2006, 2008 and 2011 are used to assist in identifying and monitoring progress in the selected sites. The identified temporal land cover enables setting the model parameters and validation of the model predictions. It is clear from this introduction that a pressing challenge for present and future modeling of vegetation propagation lies in finding sound approaches to develop strategies for data exploration and modeling. Other problems are associated with the implementation of any approach employing limited data. In the absence of appropriate data, the best alternative currently available for setting model parameters and validation is the use of the ecological principles and indirectly known rules. A new approach has to be proposed entailing coupling of dynamic and spatial models.

\section{Methods}

A new method has been developed to provide spatio-temporal modelling of vegetation propagation across a disturbed space linked to GIS. Vegetation productivity is evaluated by classification of a series of three aerial images for each disturbed site. In dependence on the quality of captured aerial images and processing outputs, the obtained results have to be adjusted in the GIS for subsequent processing by modeling tools. Satellite data and automatic classification methods can be used for the described tasks as an alternative to the captured aerial images. However, digital image processing techniques dedicated to high resolution data, such as multi-temporal Landsat TM [10], have limited potential because very high resolution images are needed to provide more detailed information at the level of individual trees and plant communities. As an alternative to selected classification methods for vegetation mapping, object-based image analysis can efficiently process a large volume of very high-resolution image data, but requires advanced software tools and time-consuming testing [11].

Following data conversion, final classified datasets are used as inputs for the proposed spatio-temporal model (Fig. 1). The original resolution of aerial images of approx. 50 centimeters was resampled to a resolution of 5 meters in order to decrease the number of cells in the grid for more efficient and faster simulations. Several levels of vegetation density for modeling vegetation propagation across a disturbed space have been evaluated: high, middle, low and no vegetation. The classified datasets originated from aerial images captured in 2006 are used to set the initial vegetation density on a relative scale from 0 (no vegetation) to 1 (long-term maximum vegetation density). High density is represented by a value of 0.90 , which is near the maximum long-term vegetation density. Medium density is set at a value of 0.50 . Low density is represented by the value 0.10 , which is near the minimum vegetation density. No vegetation is assigned a vegetation density value of 0.01 to simulate potential growth ability in the future. The approximate 
estimates of the vegetation density are adapted to the results of the classification methods and to the prediction abilities of the proposed spatio-temporal model. The image datasets originating from aerial images captured in 2008 and 2011 are used to estimate the growth parameters in the basic logistic growth term of the spatio-temporal model. The numbers of cells attached to the defined levels of the vegetation density for the former pyrite ore mine and for the ash deposits of the power station are depicted in final classified datasets (Fig. 1).

The growth and propagation of vegetation are described by a set of ordinary differential equations, which are attached to the grid of cells. The time change in the vegetation density in a cell located in the $i^{\text {th }}$ row and the $j^{\text {th }}$ column is assembled as the sum of the local vegetation growth, the vegetation propagation from neighbor cells and the effects of outlying vegetation propagation:

$$
\begin{gathered}
\frac{d x_{(t) i, j}}{d t}=r_{(t) i, j} x_{(t) i, j}\left[1-\frac{x_{(t-d 1) i, j}}{K_{(t) i, j}}\right]+ \\
+k_{d 2}\left[x_{(t-d 2) i-1, j}+x_{(t-d 2) i+1, j}+x_{(t-d 2) i, j-1}\right. \\
\left.+x_{(t-d 2) i, j+1}-4 x_{(t-d 2) i, j}\right]+ \\
+k_{d 3}\left[1-e^{\left.k_{e 3} \sum_{i, j} x_{(t-d 3) i, j}\right]}\right.
\end{gathered}
$$

The local vegetation growth in each cell is controlled by the growth ratio $r_{(t) i, j}$ and by the long-term maximum vegetation density $K_{(t) i, j}$. The time delay $d_{1}$ is used to include the effects of delayed response from the former vegetation age groups in each cell. This includes increasing the vegetation density by seeding processes and by clonal propagation. Spreading of vegetation from/to neighbor cells depends on the gradients between the local cell and its neighbor cells. The level is adjusted by coefficient $k_{d 2}$. The time delay $d_{2}$ sets the delayed effects of vegetation propagation from/to neighbor cells. Vegetation propagation by the effects of outlying vegetation propagation is represented by the last term in Eq. (1). The intensity of vegetation propagation across the whole area of interest is controlled by setting coefficients $k_{d 3}$ and $k_{e 3}$. The time delay $d_{3}$ sets the delayed effects of vegetation propagation across the whole area of interest.

The initial conditions of a set of ordinary differential equations in the spatio-temporal model are based on vegetation densities estimated from the 2006 aerial image. For each selected site, this is given by the equation:

$$
x_{(t=0) i, j}=x_{(2006) i, j}
$$

where the value of $x_{(2006) i, j}$ is: 0.90 for the high vegetation density, 0.50 for the middle vegetation density and 0.10 for the low vegetation density. If no vegetation was detected, the initial value is set at 0.01 . The setting of the growth ratio $r_{(t) i, j}$ for each cell originates from the estimate of parameter $r$ in the equation for logistic growth [12]:

$$
x_{(k) i, j}=\frac{c_{i, j}}{1+e^{a_{i, j}-r_{i, j} t(k)}},
$$

where $k=1,2$ and 3 for years 2006, 2008 and 2011, respectively. For example, an estimate of growth ratio $r$ for values $x_{2006}=0.10, x_{2008}=0.50, x_{2011}=0.90$ and the long-term maximum vegetation density (carrying capacity) $c=1.00$ is: $r=0.87$ ( $a=2.02)$.

There is also stagnation of vegetation density or even a decrease in the vegetation density due to the local working activities in some parts of the two selected sites. Thus, the growth ratio can be zero or even have a negative value. For final predictions focused on the various effects of vegetation propagation, the values of the growth ratio $r$ and other coefficients must be revised and balanced in order to adjust the model predictions to the terrain data. The numerical solution of a spatio-temporal model represents a part of data processing outside of the GIS. The numerical solution is based on the MATLAB solver for DDEs (delay differential equations) with constant delays.

\section{Results and Discussion}

The simulation outputs are tested for a few selected case-oriented studies. The first case study can simulate vegetation growing without any neighbor-neighbor 
Site of a former pyrite ore mine
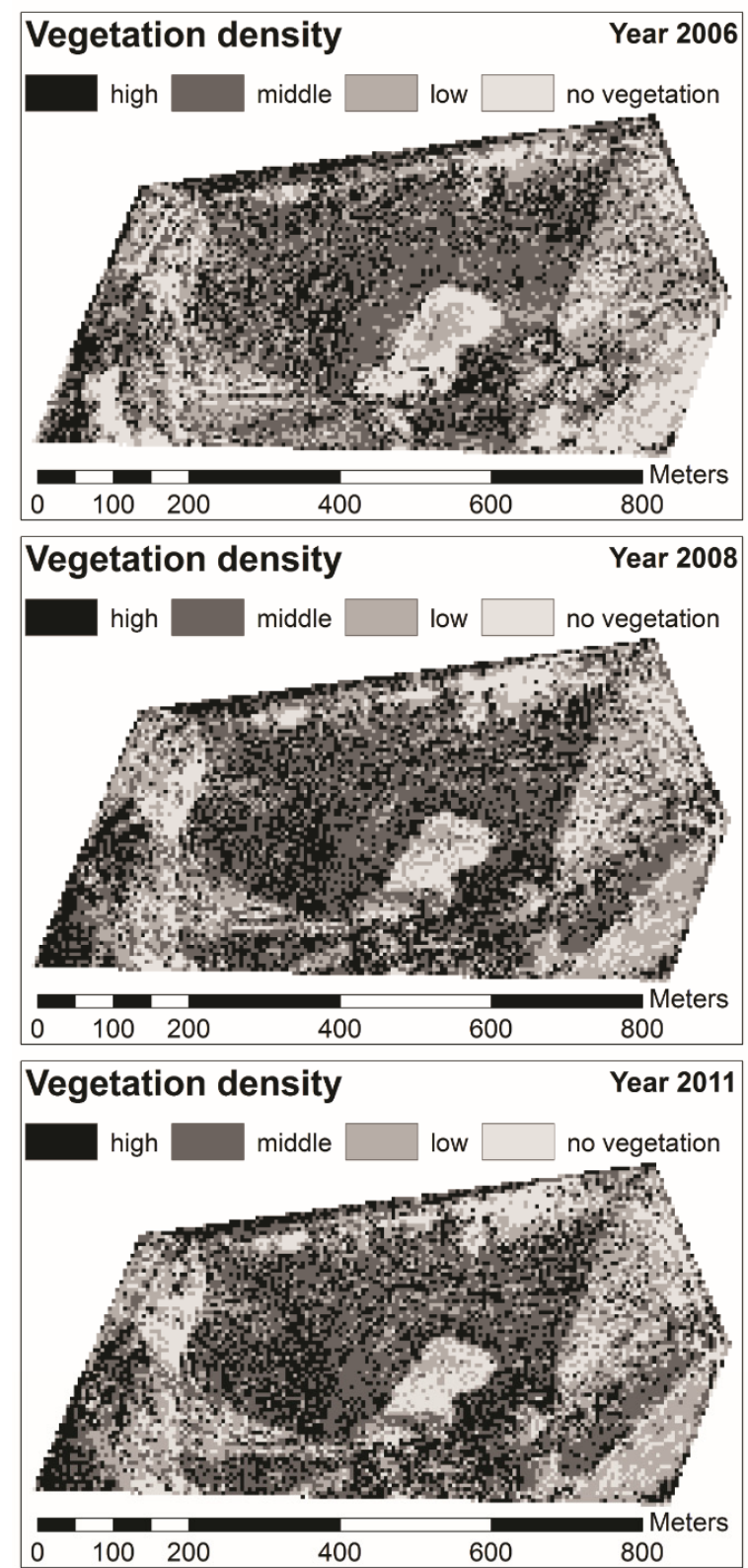

Vegetation density: Estimated number of cells

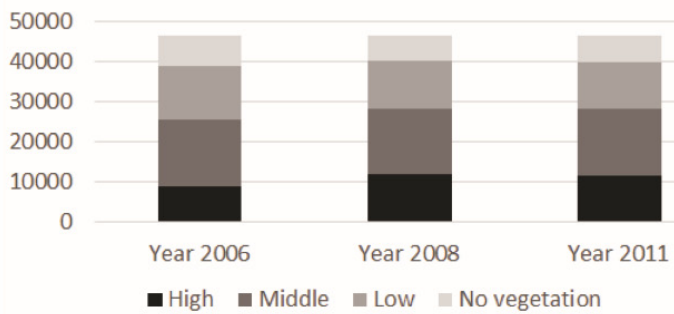

Site of an ash deposits of the power station
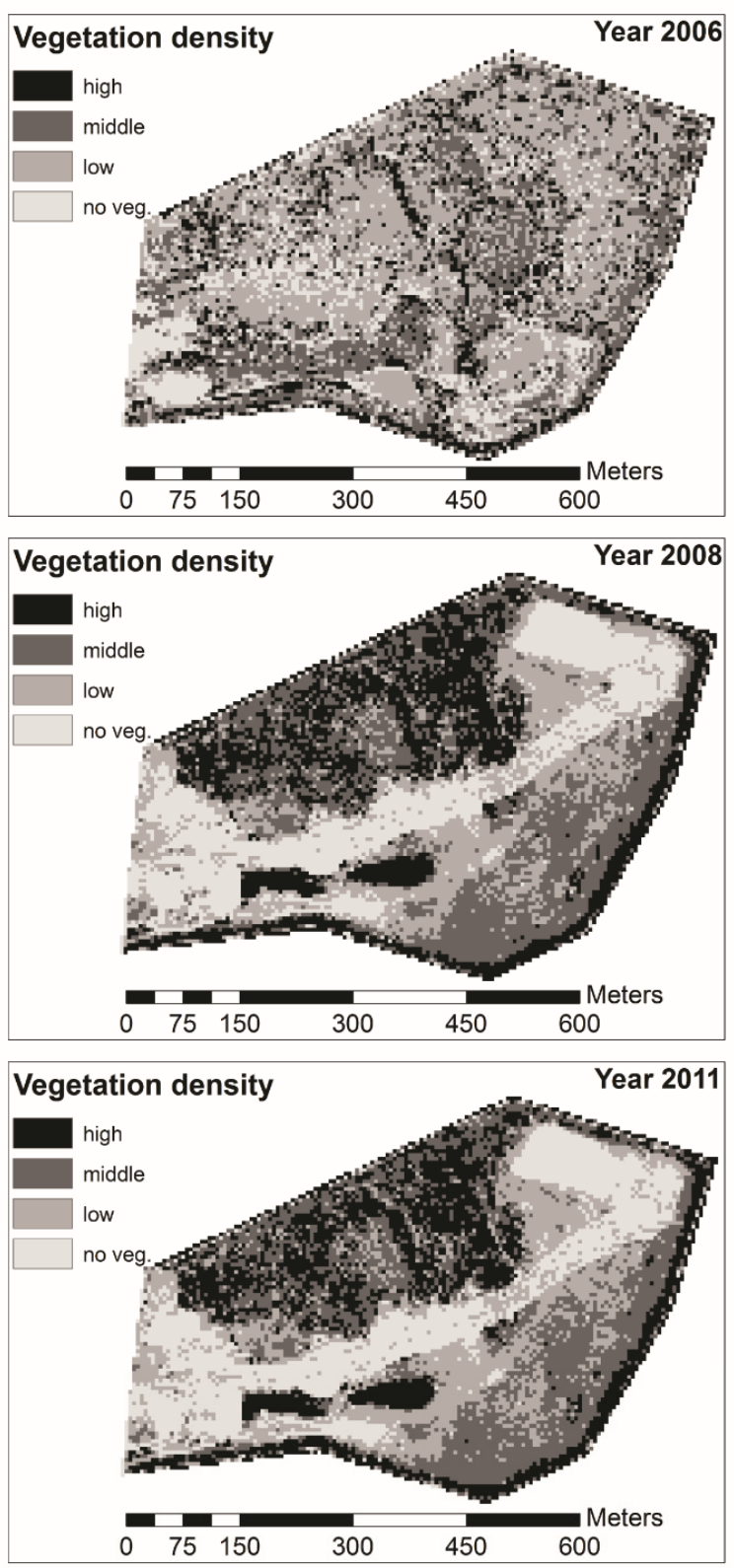

Vegetation density: Estimated number of cells

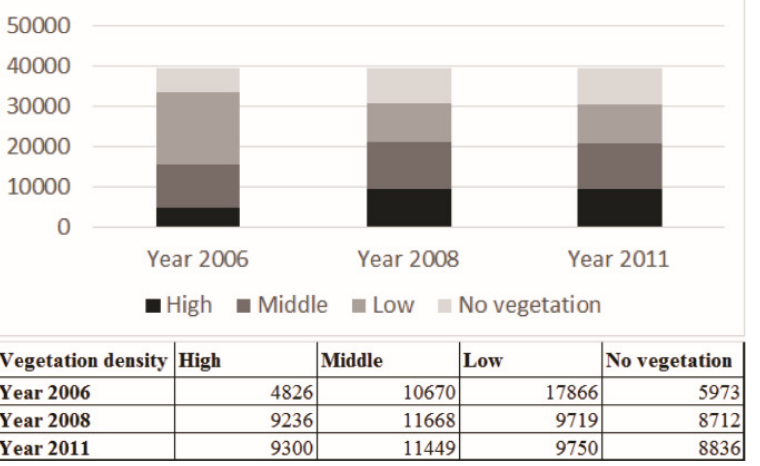

\begin{tabular}{|c|c|c|c|c|c|c|c|c|c|}
\hline Vegetation density & High & Middle & Low & No vegetation & Vegetation density & High & Middle & Low & No vegetation \\
\hline Year 2006 & 8841 & 16438 & 13260 & 7720 & Year 2006 & 4826 & 10670 & 17866 & 5973 \\
\hline Year 2008 & 11742 & 16381 & 11792 & 6344 & Year 2008 & 9236 & 11668 & 9719 & 8712 \\
\hline Year 2011 & 11439 & 16485 & 11922 & 6413 & Year 2011 & 9300 & 11449 & 9750 & 8836 \\
\hline
\end{tabular}

Fig. 1 Experimental data: Image datasets represented by two selected sites with images captured in 2006, 2008 and 2011. 
interactions and delayed variables for both the sites. The second and the third case studies for both the sites extend the previous study to include local vegetation propagation and the effects of outlying vegetation propagation, respectively. The final fourth case study in addition to the previous case studies explores the delayed impacts in the logistic growth term and the delayed outcome by vegetation propagation across the disturbed space. Computer based modeling of vegetation propagation for both the selected sites illustrated by a processing schema (Fig. 2).

The final image datasets are adjusted and stored at 5 meter resolution in order to balance local disturbances and to decrease the number of cells for more efficient simulation runs of the spatio-temporal modeling. Thus, the spatio-temporal model for the former pyrite ore mine and the ash deposits of the power station is represented by sets of $185 \times 345$ and $212 \times 266$ ordinary differential equations, respectively. Manual correction of the classified datasets was finally used instead of various techniques of supervised classification that were more sensitive to individual aerial images and to setting of training samples. For very high resolution satellite images in an explored period, there was an incomplete range of images in the archive database for classification or clustering by a number of other techniques, such as vegetation indexes, etc. [6].

The classified image datasets originate from the years: 2006, 2008 and 2011. These datasets are stored in the GIS and exported to the BSQ (band sequential) scheme for storing pixel values of images in a file. The BSQ schemes are selected in order to provide integration of the model inputs, classified images, and predictions of vegetation density at the defined time points in the framework of the data cube [13]. The data cubes mainly used in a data mining approach turned out to be an efficient scheme for the described spatio-temporal modeling that deals with data management of large image datasets. The classified image datasets and model predictions are stored in a data cube with two dimensions formed by the $(x)$ and (y) spatial axis of the image display and the third (z) formed by the time of dataset acquisition or prediction. In addition to a data cube file, it is necessary to load a header file with setting of the data cube dimensions and extra information about the time points of image dataset acquisition, as well as time points of the attached model predictions in the simulation period.

The input image datasets for the spatio-temporal model represent two selected sites in different environments, the abandoned sedimentation basin of a former pyrite ore mine, and the ash deposits of a power station. For the investigated period (2006-2011) and available aerial images, the first site indicates sparse human activities whereas some parts of the second site were highly disturbed during the whole investigated period. The classified image datasets for the ore mine indicate an increase in the number of cells classified as high-density vegetation to the detriment of cells classified as no vegetation or low-density vegetation. For the ash deposits, there is an increase in the number of cells classified as high-density vegetation to the detriment of the cells classified as low-density vegetation. The increase in the number of cells classified as no vegetation is related to the human.

The proposed spatio-temporal model deals with prediction of the vegetation density for each cell $(5 \times 5$ meters). The grid sizes are $185 \times 345$ and $212 \times 216$ for the former pyrite ore mine and the ash deposits of the power station, respectively. The original models described by many authors were extended to include delayed variables, local vegetation propagation between each cell and its neighboring cells and by the effects of outlying vegetation propagation across the whole area.

These phenomena are tested on two selected sites. The initial estimate of the growth ratio $r$ for the logistic growth is based on a simplified assumption of the first case study that is focused on the logistic growth with no delayed variables and no propagation effects. Instead of various generalized versions of the logistic 


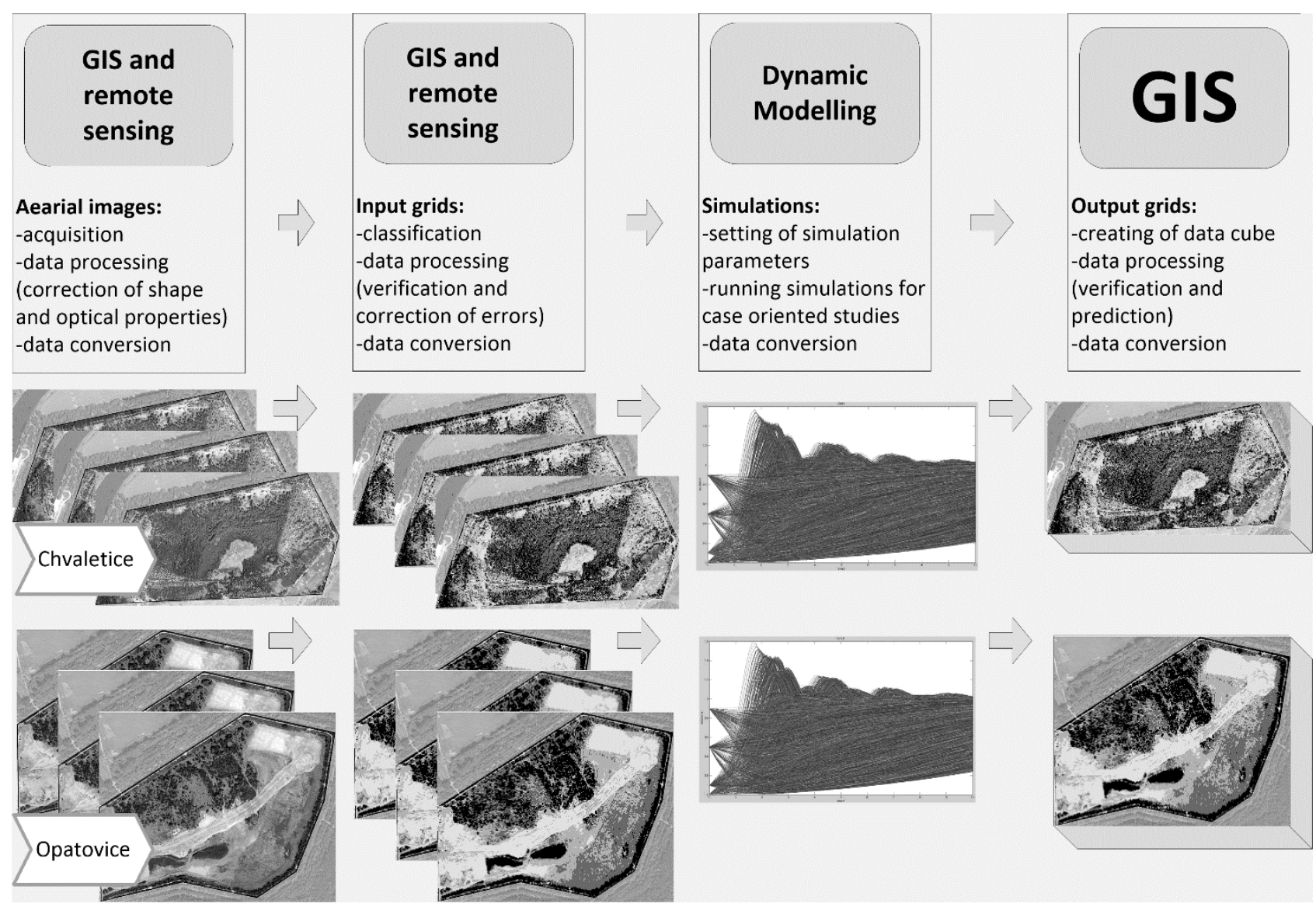

Fig. 2 Computer based modeling of vegetation propagation linked to GIS for both the selected sites based on individual steps, such as processing of primary data and classifications by software tools for remote sensing, preprocessing of model input data in the GIS, numerical solution in MATLAB and post-processing of the simulation results for presentation of various case-oriented studies.

growth [14], a basic form is used to test proposed extensions in the next case-oriented studies. Only three available image datasets could be used to estimate growth ratio $r$ in each cell in the grids of both the selected sites. To provide an estimate and to test the influence of various growth ratios $r$ in all the cells, the cells classified as having no vegetation are assigned an initial vegetation density of 0.01. As shown in the histograms (Fig. 1), some cells indicate a decrease in the vegetation density in the subsequent periods due to the local human activities. This leads to negative values of growth ratio $r$.

In order to test a proposed numerical model based on the DDE solver in MATLAB, the first case study is calculated without delayed variables and without the effects of vegetation propagation. The initial vegetation densities are set at the levels: 0.90 (high density), 0.5 (middle density), 0.1 (low density) and 0.01 (no vegetation). Thus, the changes in the vegetation density are dependent only on the estimates of the growth ratio, $r$. Positive values of $r$ result in increasing vegetation densities to the long-term maximum in correspondence with the anticipated revitalization. Negative values of $r$ cause a decrease in the vegetation density, which corresponds to short-term human activities in some parts of the areas of interest, mainly the ash deposits of the power station. After verification of the spatio-temporal model and putting into operation the data exchange with a data cube scheme in the framework of the first case study, extended studies were started to explore the effects of the local vegetation propagation, of outlying vegetation 
propagation and of delayed variables (Fig. 2).

\section{Conclusions}

The presented spatio-temporal model focused on vegetation propagation across a disturbed space linked to GIS was developed to predict the vegetation density in two disturbed sites. Based on the described case studies, setting of the growth and the propagation coefficients enables us to use the spatio-temporal model for a number of similar sites. Using a data cube schema for storage of image datasets offers high flexibility for data exchange among the model solvers, GIS and other software tools. Numerical solutions of larger grids are limited by the power of the computing tools. It is anticipated that the use of modern computer systems based on parallel computing and new advanced remote sensing technology will bring new insights into the studied processes of vegetation propagation. The created GIS project makes it possible to complement the output image datasets by a wide range of environmental data that can be used for decision-making processes in order to optimize remediation processes at selected sites and similar areas of interest.

\section{Acknowledgements}

The spatio-temporal model and the GIS project have been processed using MATLAB, ArcGIS and ENVI in the GIS Laboratory of the Faculty of Science, Charles University in Prague.

\section{References}

[1] Tilman, D., and Kareiva, P. 1997. Spatial Ecology: The Role of Space in Population Dynamics and Interspecific Interaction. 1st ed. Princeton: Princeton University Press.

[2] Shigesada, N., and Kawasaki, K. 1997. Biological
Invasions: Theory and Practice. 1st ed. Oxford: Oxford University Press.

[3] Okubo, A. 1980. Diffusion and Ecological Problems: Mathematical Models. 1st ed. Berlin: Springer-Verlag.

[4] Breckling, B., Müller, F., Reuter, H., Hölker, F., and Fränzle, O. 2005. "Emergent Properties in Individual-Based Ecological Models-Introducing Case Studies in an Ecosystem Research Context." Ecological Modelling 186: 376-88.

[5] Colasanti, R. L., Hunt, R., and Watrud, L. 2007. "A Simple Cellular Automaton Model for High-Level Vegetation Dynamics.” Ecological Modelling 203: 363-74.

[6] Erener, A. 2011. "Remote Sensing of Vegetation Health for Reclaimed Areas of Seyitömer Open Cast Coal Mine." International Journal of Coal Geology 86: 20-6.

[7] Perpina, C., Martínez-Llario, J. C., and Pérez-Navarro Á. 2013. "Multicriteria Assessment in GIS Environments for Siting Biomass Plants." Land Use Policy 31: 326-35.

[8] Zeiler, M. 2010. Modeling Our World: The ERSI Guide to Geodatabase Concepts. Redlands, California: ESRI Press.

[9] Bryndova, I., and Kovar, P. 2004. "Dynamics of The Demographic Parameters of the Clonal Plant Calamagrostis Epigejos (L.) Roth in Two Kinds of Industrial Deposits (Abandoned Sedimentation Basins in Bukovina and Chvaletice)." In Natural Recovery of Human-Made Deposits in Landscape, edited by Kovar, P. Prague: Academia, 267-76.

[10] Prakash, A., and Gupta, R. P. 1998. "Land-Use Mapping and Change Detection in a Coal Mining Area-A Case Study in the Jharia Coalfield, India." International Journal of Remote Sensing 19 (3): 391-410.

[11] Bauer, Th., and Strauss, P. 2014. "A Rule-Based Image Analysis Approach for Calculating Residues and Vegetation Cover under Field Conditions." Catena 113: 363-9.

[12] Krebs, C. 1994. Ecology: The Experimental Analysis of Distribution and Abundance. 4th ed. Harper Collins College Publishers, 198-211.

[13] Usman, M., Pears, R., and Fong, A. C. M. 2013. “A Data Mining Approach to Knowledge Discovery from Multidimensional.” Knowledge-Based Systems 40: 36-49.

[14] Birch, C. P. D. 1999. "A New Generalized Logistic Sigmoid Growth Equation Compared with the Richards Growth Equation." Annals of botany 83: 713-23. 